\title{
Xvent-2 expression in regenerating Xenopus tails
}

\author{
Elena S. Pshennikova, Anna S. Voronina \\ A.N. Bach Institute of Biochemistry, Research Center of Biotechnology RAS, Moscow, Russia \\ Correspondence to: Anna S. Voronina. A.N. Bach Institute of Biochemistry, Research Center of Biotechnology RAS, Moscow 119071, Russia. \\ Email: voronina_a@mail.ru.
}

\begin{abstract}
The tail of Xenopus tadpole is an excellent model for appendage regeneration studies. We analyzed the distribution pattern of the transcription factor Xvent-2 mRNA and protein in the beginning of the regeneration of Xenopus tadpole tail stumps after amputation. We revealed the emergence of Xvent-2 mRNA and protein in regeneration bud during the first day after amputation. The data obtained confirm that soon after amputation of the part of the Xenopus tadpole tail, there occurs the emergence of a structure, to some extend, resembling the early embryonic tail bud.
\end{abstract}

Keywords: Xenopus tadpole; Xvent-2; tail bud; regeneration

Received: 28 October 2019; Accepted: 10 July 2020; Published: 20 July 2020.

doi: $10.21037 /$ sci-2019-044

View this article at: http://dx.doi.org/10.21037/sci-2019-044

\section{Introduction}

The investigations of regeneration processes are still of grate scientific and therapeutic interest. Beside the limbs and limb buds, tadpole tails serve an adequate model for organs regeneration studies, because they consist of many tissues formed by almost all the cell lineages $(1,2)$. It is known that about $75 \%$ distal part of the tail can be cut off without threatening the animal, and in 10 to 14 days a fullsize tail will successfully regenerate. It is of interest for the proximal $60 \%$ part of the tail is known to be originated from the trunk, not from the tail bud (1). One can expect that the cut tail regeneration is ensured by the mechanisms which provide the embryonic development, and so some works were devoted to the comparison of the developing and regenerating tails. It was shown that the $M s x$ genes and the genes involved in Notch signaling, were expressed in the same way in tail development and regeneration $(1,2)$. The $M s x$ genes coding for transcriptional repressors, active downstream the Bmp cascade, are probably involved in processes of muscle cells dedifferentiation taking place during urodele appendage regeneration. Some genes, e.g., Hox genes and genes for the fibroblast growth factor-2, were important in development. There were described differences in genes activity during tail regeneration and development. These are Chordin and Noggin1, the Bmp cascade inhibitors, which were revealed in the developing tail bud but not in the regenerating tails. The morphogen sonic hedgehog (shh) gene activity, characteristic for the neural tube in development, also was not revealed in regenerates, probably because they may have some defects in spinal cord patterning. Furthermore, it was shown that the neural crest cells, arising from the neural plate border and then migrating away, did not participate in the origin of the replaced melanophores. The last originate from existent precursors harbored in blastema and earlier recruited from tissues close to the stump $(1,2)$. To learn the molecular mechanisms in detail, a transcriptional analysis using RNA-Seq, of the first 72 hours of the $X$. tropicalis tails regeneration process was performed (3). According to their ontology and patterns of expression during regeneration, all the genes studied, were divided into 8 clusters (3).

The most comprehensive investigation of the rates of transcription and cell-type shifts that happen in the process of regeneration, was performed just recently (4). Using the high-throughput single cell RNA sequencing method, there were analyzed $X$. laevis tadpole tails at different times after amputation, regeneration-competent and incompetent tadpoles, and also intact tails at appropriate stages of development. Monitoring the data range, the authors revealed that the most remarkable cell-type shifts during the regeneration concerned a previously unexplored 
epidermal cells, which they designated as the regeneration organizing cells (ROCs). Insofar as this cell population specified the reaction to amputation of the regenerationcompetent tadpoles only, and expressed genes that ensured regeneration, e.g., Wnt5, Fgf10. Fgf20, Msx1, Bmpr1a, it was proposed that the ROCs may serve as significant contributor to regenerative reaction. These data suggested the ROCs to be a signaling center that produce the agents to promote progenitor proliferation in many adjacent tissues. This rise in proliferation rates also elucidates why the lost tail tissues recover by their progenitors and do not require the new emergent multipotent cell states (4).

Earlier we hypothesized the cells producing the Xvent-2 to belong to neural crest descendants $(5,6)$. In ontogenesis, the neural crest formation can be divided into several stages, when the embryonic cells are sequentially affected by certain modules of regulatory factors. The first-level signals, which function at the gastrula stage, are ensured by Wnt, BMP, and FGF molecules. The combined action of inductive signals leads to enhanced expression of the module of genes encoding the second-level transcription factors $D l x, M s x, Z i c$, and Pax3/7. The third level is characterized by the transcription factors Snail1/2, AP-2, FoxD3, Twist, $I d, c M y c$, and Sox9/10, which start their expression before the migration of neural crest cells $(7,8)$. There's a lot of evidence that the migrating cells of the neural crest retain pluripotency (8) and thus the neural crest is regarded as the fourth germ layer.

The Xvent-2 transcription factor is a part of a $\mathrm{Bmp} /$ Smad cascade. Being transferred to the nucleus by Smad4, it binds to promoters of some genes and so enhances or represses their transcription. Hence, the Xvent-2 expression in the neural crest cells may be quite reasonable. Moreover, most likely the Xvent-2 protein is not only an indicator of the gastrula pluripotent cells, but is characteristic for the pluripotent and multipotent cells during further development $(5,6)$. In particular, in Xenopus tadpole tails it was revealed in mesenchyme, around the neural tube and notochord, in between somites, between somites and epidermis, also in fins. Such allocation coincides with routs of the neural crest cells migration during the embryo development $(5,9)$. Some kind of pluripotent cells were shown to present in tail buds, which generate the proximal part of the body, including caudal axial structures: the caudal neural tube, tail gut and, probably, the notochord (10).

Here we show the expression patterns of the Xvent-2 mRNA and protein in the Xenopus tadpole tail in the first hours after amputation.

\section{Methods}

Xenopus laevis tadpoles at the stage 45, according to Nieuwkoop and Faber (11), had their tails amputated at a position approximately $1 / 2$ the length. After that, the tadpoles were returned to container with fresh water at $14^{\circ} \mathrm{C}$ and analyzed at 0, 6 and 20 hours post amputation.

Rabbit polyclonal antibodies against Xenopus recombinant Xvent-2 were obtained as described in $(5,6)$. The recombinant protein was verified by mass spectrometry, and the spectra were compared to theoretical predictions with the use of the MS-FIT program (http://prospector. ucsf.edu). The recXvent-2 protein was concentrated by methanol precipitation, dissolved in phosphate buffer and used to raise polyclonal antiserum in rabbits (5). For wholemount immunostaining anti-reXvent-2 rabbit antibodies were purified and conjugated to horseradish peroxidase (IMTEK, Moscow).

Whole-mount immunostaining of the Xenopus embryos was carried out as described earlier (5).

Whole-mount in situ hybridization was performed by a standard procedure (12). Plasmid pRN3carrying Xvent-2 cDNA was used to obtain the digoxigenine-(+ and -) RNA by T3 or T7 RNA polymerase (Roche; Fermentas). All animal manipulations complied to European directive 2010/63, under the control of the veterinary services of Paris (authorization \#75-419).

\section{Results and discussion}

We have analyzed the spatial patterns of the Xvent-2 mRNA and protein distribution in Xenopus tadpole tail stumps right after the amputation of distal tail parts, and during the first day of regeneration (Figure 1).

In previous paper (6) we described that the Xvent-2 protein in the tail bud was detected only at the early stages of development and then it localized in mesenchyme due to its migration, or migration of the cells harboring it, along the axial structures. The same distribution is seen in (Figure $1 A, B)$. It is also seen that during the normal embryonic development, the expression of the Xvent-2 mRNA occurred only in the tail tips containing pluripotent tail bud cells (Figure 1G). Soon after amputation at the site of injury, the Xvent-2 mRNA and protein could be detected (Figure $1 C, D, E, F)$. The Xvent-2 mRNA is revealed in the wound area and in the regenerating bud, whereas in intact tadpoles, in this site of tail we could not detect it (Figure 1G).

The presence of the Xvent-2 protein in the healing 

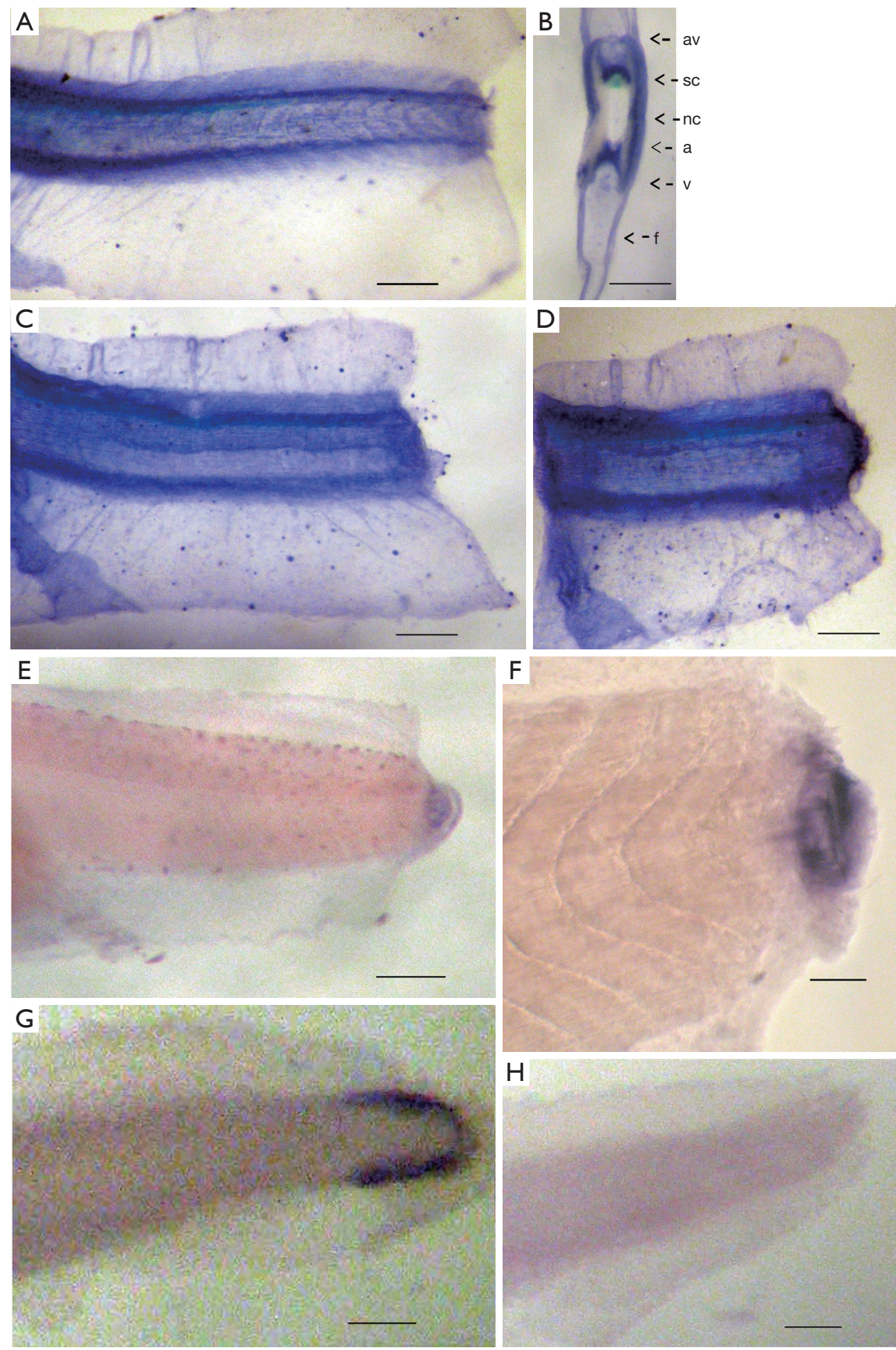

Figure 1 The spatial distribution of Xvent-2 protein and mRNA in the regenerating tails of Xenopus embryos. (A,B,C,D) Immunostaining; (E,F,G,H) in situ hybridization. A: 0 h; C: 6 h; D,E,F: 20 h after amputation; G,H: amputated tail parts; B: cross-section of the tail in the amputation area. Sections thickness about $0.2 \mathrm{~mm}$. nc, notochord; sc, spinal cord; a, dorsal aorta; v, posterior cardinal vein; av, dorsal lateral anastomosing vessel; f, fin; H, control in situ hybridization with a sense probe. Scale bars: A-E, G,H, $500 \mu \mathrm{m} ; \mathrm{F}, 100 \mu \mathrm{m}$. 
wound can be due to both synthesis de novo and the mesenchymal cells migration, probably from the neural crest to the wound. The Xvent-2 being a transcriptional factor is known to upregulate its own expression (13) and so does the BMP found in the wounds $(2,14)$.

The emergence of the Xvent-2 expression in the regeneration bud indicates the restoring there, at least partially, of the tail bud regulatory mechanisms. As it was already shown (4), the tadpole tail tissues-notochord, muscles, nerves etc.- - regenerate from their progenitors, stored in these tissues. The function of a trigger for such differentiations may be performed by the ROCs (4). Earlier it was also suggested the Xwnt-5a to play a critical role in the regenerating tail (15), but the inducer of its expression after the amputation was not clear. It is to be assumed that in the case of the tail mesenchymal cells, which apparently belong to the neural crest, the expression of several genes (the Xvent-2, in particular and probably Xwnt-5a) may be activated by the ROCs, and as a result, the regeneration bud is formed.

\section{Conclusions}

We analyzed the distribution patterns of Xvent- 2 mRNA and protein at the beginning of the regeneration of Xenopus tadpole tail stumps after amputation. We revealed the emergence of Xvent-2 mRNA and protein in regeneration buds during the first day after amputation. It is of interest insofar as before amputation there was no Xvent-2 mRNA staining detected in the zone of cutting. The data obtained demonstrate that the Xvent-2, in addition to the $M s x$ genes and the genes involved in Notch signaling $(1,2)$, was expressed in the comparable way in tail development and regeneration. It also confirms that soon after amputation of the part of the Xenopus tadpole tail, there occurs the emergence of a structure, to some extend, resembling the early embryonic tail bud.

\section{Acknowledgments}

Funding: None.

\section{Footnote}

Conflicts of Interest: Both authors have completed the ICMJE uniform disclosure form (available at http://dx.doi. org/10.21037/sci-2019-044). The authors have no conflicts of interest to declare.
Ethical Statement: The authors are accountable for all aspects of the work in ensuring that questions related to the accuracy or integrity of any part of the work are appropriately investigated and resolved. All animal manipulations complied to European directive 2010/63, under the control of the veterinary services of Paris (authorization \#75-419).

Open Access Statement: This is an Open Access article distributed in accordance with the Creative Commons Attribution-NonCommercial-NoDerivs 4.0 International License (CC BY-NC-ND 4.0), which permits the noncommercial replication and distribution of the article with the strict proviso that no changes or edits are made and the original work is properly cited (including links to both the formal publication through the relevant DOI and the license). See: https://creativecommons.org/licenses/by-nc-nd/4.0/.

\section{References}

1. Beck CW, Belmonte JCI, Christen B. Beyond Early Development: Xenopus as an Emerging Model for the Study of Regenerative Mechanisms. Dev Dyn 2009;238:1226-48.

2. Chen Y, Love NR, Amaya E. Tadpole tail regeneration in Xenopus. Biochem Soc Trans 2014;42:617-23.

3. Chang J, Baker J, Wills A. Transcriptional dynamics of tail regeneration in Xenopus tropicalis. Genesis 2017;55:e23015.

4. Aztekin C, Hiscock TW, Marioni JC, et al. Identification of a regeneration organizing cell in the Xenopus tail. Science 2019;364:653-8.

5. Pshennikova E, Goncharenko A, Voronina A. The Xvent-2 protein expression in multipotent cells of the neural crest in tails of Xenopus laevis and Rana temporaria tadpoles. Indian Journal of Fundamental and Applied Life Sciences 2014;4:180-7.

6. Pshennikova ES, Voronina AS. The proteins of Ventfamily and their mRNAs are located in different areas of the tails of Zebrafish and Xenopus embryos. Int J Biochem Cell Biol 2016;79:388-92.

7. Meulemans D, Bronner-Fraser M. Gene-regulatory interactions in neural crest evolution and development. Dev Cell 2004;7:291-9.

8. Pshennikova ES, Voronina AS. Neural Crest-An Unusual Population of Embryonic Cells. Molecular Biology (Mosk) 2019;53:256-67.

9. Theveneau E, Mayor R. Neural crest delamination and 
migration: From epithelium-to-mesenchyme transition to collective cell migration. Dev Biol 2012;366:34-54.

10. Bronner ME, LeDouarin NM. Development and evolution of the neural crest: An overview. Dev Biol 2012;366:2-9.

11. Nieuwkoop PD, Faber J. Normal Table of Xenopus laevis (Daudin): A Systematical and Chronological Survey of the Development from the FertilizedEgg till the End of Metamorphosis. 1956; Amsterdam: North Holland. Stuhlmiller and Garcia-Castro, 2012.

12. Lehmann R, Tautz D. In situ hybridization to RNA. Methods Cell Biol 1994;44:575-98.

doi: 10.21037/sci-2019-044

Cite this article as: Pshennikova ES, Voronina AS. Xvent-2 expression in regenerating Xenopus tails. Stem Cell Investig 2020;7:13.
13. Henningfeld KA, Friedle H, Rastegar S, et al. Autoregulation of Xvent-2B; direct interaction and functional cooperation of Xvent-2 and Smad1. J Biol Chem 2002;277:2097-103.

14. Ho DM, Whitman M. TGF- $\beta$ signaling is required for multiple processes during Xenopus tail regeneration. Dev Biol 2008;315:203-16.

15. Sugiura T, Tazaki A, Ueno N, et al. Xenopus Wnt-5a induces an ectopic larval tail at injured site, suggesting a crucial role for noncanonical Wnt signal in tail regeneration. Mech Dev 2009;126:56-67. 